ENCYCLOPEEDIE Encyclopédie berbère

BERBERE

$24 \mid 2001$

24 | Ida - Issamadanen

\title{
Indicateur de thème
}

(syntaxe)

\section{J. Kuningas-Autio}

\section{OpenEdition}

Journals

Édition électronique

URL : http://journals.openedition.org/encyclopedieberbere/1571

DOI : 10.4000/encyclopedieberbere.1571

ISSN : 2262-7197

\section{Éditeur}

Peeters Publishers

\section{Édition imprimée}

Date de publication : 1 octobre 2001

Pagination : 3722-3725

ISBN : 2-7449-0207-1

ISSN : 1015-7344

\section{Référence électronique}

J. Kuningas-Autio, «Indicateur de thème », Encyclopédie berbère [En ligne], 24 | 2001, document I54, mis en ligne le 01 juin 2011, consulté le 25 septembre 2020. URL : http://journals.openedition.org/ encyclopedieberbere/1571; DOI : https://doi.org/10.4000/encyclopedieberbere.1571

Ce document a été généré automatiquement le 25 septembre 2020.

(c) Tous droits réservés 


\title{
Indicateur de thème
}

\author{
(syntaxe)
}

\section{J. Kuningas-Autio}

1 L'indicateur de thème est un concept proposé par Galand (1964). Il réfère au constituant nominal à l'état libre placé en tête d'énoncé, comme par exemple tamețțutnnî) dans l'énoncé :

(1) tamețțut-nni, tura-d tabratt

"La femme (en question) a écrit une lettre"

2 L'état libre est l'un des deux états que le nom peut avoir en berbère, le deuxième étant l'état d'annexion ( $C f$. “Annexion", $E B, V$ ).

3 La virgule indique une rupture intonative entre le constituant en tête d'énoncé et la prédication ( $C f$. Intonation*). Le constituant en tête d'énoncé est ainsi détaché du prédicat. Son rôle dans la prédication est, dans cet exemple, signalé par un pronom affixe que les berbérisants appellent "indice de personne"* (ici le $t$ ).

4 Ces traits - la séparation par une rupture intonative, la reprise (éventuelle; $C f$. infra) par un pronom affixe - correspondent aux traits définitoires des constructions dites disloquées. Les énoncés à indicateur de thème du berbère ont été rapprochés des énoncés disloqués en français parlé notamment par Leguil (1992).

5 L'indicateur de thème est nettement distingué du "complément explicatif" ( $C f$. "Fonctions syntaxiques", EB, XIX), le constituant qui, à l'état d'annexion, suit le verbe conjugué, comme par exemple tmețțut dans l'énoncé suivant (2) :

(2) tura-d tmetțut -nni tabratt "elle a écrit la femme (en question) une lettre"

6 Le complément explicatif correspond à ce qui était traditionnellement considéré comme le sujet grammatical du berbère.

7 Selon la thèse classique d'André Basset (1950), ce constituant nominal antéposé devait être analysé non pas comme sujet proprement dit, mais comme l'anticipation du sujet (ou, dans d'autres cas, comme l'anticipation de régime, etc.; $C f$. ci-dessous). Les travaux de Basset montrent déjà la difficulté d'analyser le berbère en termes grammaticaux traditionnels. 
8 Aujourd'hui, le terme "indicateur de thème" n'a pas de concurrent et il est adopté par quasiment tous les berbérisants.

9 Étant donné que l'indicateur de thème est toujours à l'état libre et que c'est l'état qui permet de distinguer le complément explicatif du complément d'objet en position postverbal (dans l'énoncé canonique, le complément explicatif est distingué du complément d'objet par l'état d'annexion, le complément d'objet étant toujours à l'état libre, $C f$. exemple 2), il ne peut, au niveau formel, être associé ni à l'un ni à l'autre. Autrement dit, sa forme à elle seule ne permet pas de dire s'il se réfère à l'agent ou au patient (ou à un autre rôle sémantique) de l'énoncé qui suit. Dans l'énoncé (3) :

(3) tamețtut, ur tessi ara asteefu

"La femme n'a pas de repos" (Picard p. 136,1. 1)

10 L'indicateur de thème (tamețtut) correspond au complément explicatif de l'énoncé canonique ou, au niveau sémantique, à l'agent, mais il n'y a rien dans sa forme qui permette de l'associer à ce rôle sémantique ; en fait, sa forme amènerait plutôt à penser qu'il s'agit d'un patient (ou d'un complément d'objet).

11 Dans l'exemple (4), l'indicateur de thème correspond (sémantiquement) au patient :

Tafunast-ag(i) ur tt-yesci xhedd (Picard p. 200,1. 20)

“Cette vache, personne n'en possède de pareille"

Mais sa forme ne change pas. L'indicateur de thème de cet exemple n'est différencié par aucun signe formel (ni morphologique ni phonologique) du constituant thématisé de l'exemple (3).

12 Dans l'exemple (4), le fait que le complément explicatif (l'indéfini hedd) est explicite facilite l'interprétation de l'indicateur de thème - qui peut pas avoir la même fonction sémantique que celui-ci. Dans l'exemple (3), c'est le complément d'objet (asteefu) qui est explicite et qui guide l'interprétation.

13 C'est cette neutralisation de l'opposition qui permet à Galand (1964) de poser la fonction syntaxique d'indicateur de thème et d'abandonner la notion d'anticipation d'André Basset, différenciée selon différentes fonctions :

14 «La fonction de l'élément initial [...] est la même dans tous [les cas] et cette unité ne doit pas être masquée par des appellations multiples comme "anticipation de sujet" et "anticipation de régime", au demeurant trop précises : ni la place ni la forme [...] ne laissent prévoir la fonction du morphème qui reprendra ce nom... » (p. 41).

15 Cette capacité d'englober différents rôles sémantiques distingue l'indicateur de thème du complément explicatif. Le rôle de complément explicatif se définit par rapport au prédicat, tandis que le rôle d'indicateur de thème se définit par rapport à l'énoncé entier. Il représente "ce dont on parle", ce dont il est question dans cet énoncé.

16 Selon Galand (1964: 42), la fonction du constituant en tête d'énoncé "consiste à désigner "le sujet" au sens vulgaire du terme, c'est-à-dire ce dont va parler". S'il utilise plutôt le terme "indicateur de thème" c'est "pour éviter toute confusion avec "le sujet" des linguistes". Pour lui, "l'inconvénient" du terme "indicateur de thème" est d'inclure une définition sémantique, ce qui est pourtant " peu dangereux si l'on ne perd pas de vue les critères formels de la fonction qu'il désigne » (ibidem).

17 Chez Galand, on perçoit la réticence - typique des linguistes de l'époque - vis-à-vis de la sémantique, ce qui peut, au moins pour une part, expliquer pourquoi, malgré les 
observations parfois très fines présumant en fait l'implication des facteurs pragmatiques, Galand s'en tient au niveau purement formel.

Certains auteurs (Bentolila 1981) parlent d'“indicateur de thème absolu" dans le cas, fréquent, où le nominal antéposé au verbe n'est pas repris par un pronom affixe :

Aftis, ur Ğin dzmizm (p. 259)

Aftis, vous ne vous êtes jamais rencontrés (toi et lui)

19 Cette configuration confirme bien que l'indicateur de thème ne peut être syntaxiquement confondu avec/assimilé à la fonction nominale primaire correspondante et que seuls les indices sémantico-pragmatiques permettent un décodage correct de l'énoncé, comme le montre aussi cette paire d'énoncés kabyles :

(6a) aksum, cciү-t = viande, j'ai mangé-la = "La viande, je l'ai mangée" (6b) aksum, cciץ viande, j'ai mangé = "De la viande, j'en ai mangé"

La présence d'un pronom affixe de reprise n'est donc pas essentielle à l'existence de la fonction indicateur de thème.

21 L'analyse des énoncés à indicateur de thème bénéficierait certainement d'une approche qui parte du discours - des différents contextes du discours dans lesquels un énoncé peut être utilisé. Ce type d'analyse permettrait en premier lieu d'expliciter les fonctions de l'énoncé à indicateur de thème dans le discours, et de le différencier fonctionnellement des énoncés canoniques; en second lieu, de définir avec plus de précision la fonction "indicateur de thème". Il a été montré par rapport aux constructions disloquées dans d'autres langues, en particulier en français parlé ( $C f$. Lambrecht 1981), qu'une démarche partant des unités minimales n'est pas suffisante, et qu'elle doit être complétée par une démarche inverse - une démarche allant du haut (du discours) vers le bas (i.e. vers les unités minimales). Ce type d'analyse permettrait d'expliquer en quoi l'indicateur de thème est un thème et non pas un sujet par exemple, pourquoi un énoncé berbère se définit-il mieux en termes de "thème" (ou d'"indicateur de thème") et de "complément explicatif" qu'en termes de catégories grammaticales traditionnelles.

\section{BIBLIOGRAPHIE}

BASSET A., "Sur l'anticipation en berbère”. Mélanges William Marçais, Maisonneuve (Paris), p. 17-21

[repris dans Articles de dialectologie berbère, Paris, Klincksieck (SLP), 1959, p. 90-100].

BENTOLILA F., Grammaire fonctionnelle d'un parler berbère - Aït Seghrouchen d'Oum Jeniba (Maroc),

SELAF, Paris, 1981.

CHAKER S., Un parler berbère d'Algérie (Kabylie) : syntaxe, Université de Provence, 1983/a.

CHAKER S., "Le problème des catégories syntaxiques en berbère", Travaux du Cercle Linguistique

d'Aix-en-Provence, I (Les parties du discours), 1983/b, 39-59.

CHAKER S., Textes en linguistique berbère (introduction au domaine berbère), CNRS, Paris,1984. 
CHAKER S., Linguistique berbère. Etudes de syntaxe et de diachronie, Peeters, Paris/Louvain, 1995.

CHAKER S., "Quelques faits de grammaticalisation dans le système verbal

berbère".Grammaticalisation et reconstruction. Mémoires de la Société de Linguistique de Paris, Nouvelle série, tome V, Klincksieck, 1996, p. 103-121.

GALAND L., Un cas particulier de phrase non verbale : "l'anticipation renforcée" et l'interrogation en berbère, Mémorial André Basset, A. Maisonneuve, Paris, 1957.

GALAND L., L'énoncé verbal en berbère. Étude de fonctions, Cahiers Ferdinand de Saussure, 21, 1964.

GALAND L., La construction du nom complément de nom en berbère, GLECS, X., 1966

GALAND L., Types d'expansions nominales en berbère, Cahiers Ferdinand de Saussure, 25, 1969.

LAMBRECHT K., Topic, Antitopic and Verb Agreement in Non-Standard French, Pragmatics and Beyond II, 6. John Benjamins B.V., Amsterdam / Philadelphia, 1981.

LEGUIL A., Structures prédicatives en berbère, L'Harmattan, Paris, 1992.

PENCHOEN Th. G., Étude syntaxique d'un parler berbère (Aït Frah de l'Aurès), Istituto Universitario Orientale di Napoli, 1973.

PICARD A., De quelques faits de stylistique dans le parler des Irjen (Kabylie, Algérie). De la phrase inorganique à la phrase organisée, La Typo-Litho/J. Carbonnel, Alger, 1960.

PICARD A., Textes berbères dans le parler des Irjen (Kabylie-Algérie), tomes I \& II, Publications de l'Institut d'Études Orientales d'Alger XVIII, 1960.

TOURATIER C, Sujet et extraposition en berbère, BSLP, 81/1, 1986.

\section{INDEX}

Mots-clés : Linguistique 